\title{
Predictive Flavor Symmetries of the Neutrino Mass Matrix
}

\author{
M. Hirsch, ${ }^{1, *}$ Anjan S. Joshipura, ${ }^{1,2, \dagger}$ S. Kaneko, ${ }^{1, *}$ and J. W. F. Valle ${ }^{1, \S}$ \\ ${ }^{1}$ AHEP Group, Institut de Física Corpuscular-C.S.I.C./Universitat de València, Edificio Institutos de Paterna, \\ Apt 22085, E-46071 Valencia, Spain \\ ${ }^{2}$ Theoretical Physics Group, Physical Research Laboratory, Navrangpura, Ahmedabad 380 009, India
}

(Received 26 March 2007; published 12 October 2007)

\begin{abstract}
Here we propose an $A_{4}$ flavor symmetry model that implies a lower bound on the neutrinoless double beta decay rate, corresponding to an effective mass parameter $M_{e e} \gtrsim 0.03 \mathrm{eV}$, and a direct correlation between the expected magnitude of $C P$ violation in neutrino oscillations and the value of $\sin ^{2} \theta_{13}$, as well as a nearly maximal $C P$ phase $\delta$.
\end{abstract}

DOI: $10.1103 /$ PhysRevLett.99.151802

PACS numbers: 14.60.Pq, 11.30.Hv, 14.80.Cp

Unless flavor symmetries are assumed, particle masses and mixings are generally undetermined in gauge theories. Understanding mass and mixing constitutes one of the biggest challenges in elementary particle physics. Current observations do not determine all elements of the effective neutrino mass matrix $\mathcal{M}_{\nu}$ completely, and this will be a great challenge even for future experiments. Therefore theoretical ideas restricting the structure of $\mathcal{M}_{\nu}$ are needed in order to guide future searches. One such input studied extensively is the assumption that some entries in the neutrino mass matrix vanish [1]. While the phenomenological implications of the assumed zeros in the texture of $\mathcal{M}_{\nu}$ are straightforward to derive [2], it is a nontrivial task to produce a good symmetry leading to such zeros and a diagonal charged lepton mass matrix simultaneously. Although for any desired texture structure of the mass matrices such a symmetry is in principle always present, this symmetry and the associated Higgs content are sometimes discouragingly complex [3].

Here we propose a predictive flavor symmetry for leptons based on a relatively small and simple flavor group, namely $A_{4}$ or its $Z_{3}$ subgroup, and briefly analyze its phenomenological implications. We show how this provides a simple means of understanding some of the twozero textures of $\mathcal{M}_{\nu}$ studied earlier [2].

The discrete group $A_{4}$ is a 12 element group consisting of even permutations among four objects. The group is small enough to lead to a simple model but large enough to give interesting predictions. The distinguishing feature of $A_{4}$ compared to other smaller discrete groups is the presence of a three-dimensional irreducible representation appropriate to describe the three generations. This has been exploited in a number of variants. Originally, the $A_{4}$ was proposed [4,5] for understanding degenerate neutrino spectrum with nearly maximal atmospheric neutrino mixing angle. More recently, predictions for the solar neutrino mixing angle have also been incorporated in so-called tribi-maximal [6] neutrino mixing schemes [7-12]. There also exist attempts at unified $A_{4}$ models [13]. The resulting models, however, are not always simple and usually re- quire many Higgs fields. Here we show that a very simple model based on $A_{4}$ leads to two-zero textures for $\mathcal{M}_{\nu}$.

The lepton doublets $L_{i}$ are assigned as the triplet representation in all the $A_{4}$ models proposed so far. Here we propose the opposite assignment indicated in Table I, where the $L_{i}$ are assigned to the $1,1^{\prime}, 1^{\prime \prime}$ representations. The $l_{R i}$ as well as the Higgs doublets responsible for lepton masses transform as $A_{4}$ triplets, while the (undisplayed) quarks and the $S U(2)$ Higgs doublet that gives their masses are all singlets under $A_{4}$. This leads to the following terms responsible for the lepton masses:

$$
\begin{aligned}
-\mathcal{L}= & h_{1} \bar{L}_{1}\left(l_{R} \Phi\right)_{1}+h_{2} \bar{L}_{2}\left(l_{R} \Phi\right)_{1}^{\prime}+h_{3} \bar{L}_{3}\left(l_{R} \Phi\right)_{1}^{\prime \prime} \\
& +h_{1 D} \bar{L}_{1}\left(\nu_{R} \Phi\right)_{1}+h_{2 D} \bar{L}_{2}\left(\nu_{R} \Phi\right)_{1}^{\prime} \\
& +h_{3 D} \bar{L}_{3}\left(\nu_{R} \Phi\right)_{1}^{\prime \prime}+\frac{M}{2} \nu_{R i}^{T} C \nu_{R i}+\text { H.c. },
\end{aligned}
$$

where the quantities in parenthesis denote products of two $A_{4}$-triplets $l_{R}$ (or $\nu_{R}$ ) and $\Phi$ forming the representations 1 , $1^{\prime}, 1^{\prime \prime}$, respectively. Note that Eq. (1) includes the most general terms allowed by the symmetry and field content in Table I. Hence, in contrast to many other $A_{4}$ models, here one does not need to impose any additional symmetry to forbid unwanted terms.

Earlier studies on $A_{4}$ have shown that it is possible to obtain a minimum of the Higgs potential with equal vacuum expectation values (VEVs) [4]

$$
\left\langle\Phi_{1}^{0}\right\rangle=\left\langle\Phi_{2}^{0}\right\rangle=\left\langle\Phi_{3}^{0}\right\rangle \equiv \frac{v}{\sqrt{3}} .
$$

This minimum leads to charged lepton and Dirac neutrino mass matrices $M_{l}$ and $m_{D}$ given by, respectively

TABLE I. Lepton multiplet structure of the model.

\begin{tabular}{lrrrrrrc}
\hline \hline & $L_{1}$ & $L_{2}$ & $L_{3}$ & $l_{R i}$ & $\nu_{R i}$ & $\Phi_{i}$ & $\Delta$ \\
\hline$S U(2)$ & 2 & 2 & 2 & 1 & 1 & 2 & 3 \\
$U(1)$ & -1 & -1 & -1 & -2 & 0 & 1 & 2 \\
$A_{4}$ & 1 & $1^{\prime}$ & $1^{\prime \prime}$ & 3 & 3 & 3 & $1^{\prime}$ or $1^{\prime \prime}$ \\
\hline \hline
\end{tabular}




$$
\begin{aligned}
& M_{l}=v \operatorname{diag}\left(h_{1}, h_{2}, h_{3}\right) U \\
& m_{D}=v \operatorname{diag}\left(h_{1 D}, h_{2 D}, h_{3 D}\right) U,
\end{aligned}
$$

with

$$
U=\frac{1}{\sqrt{3}}\left(\begin{array}{ccc}
1 & 1 & 1 \\
1 & \omega & \omega^{2} \\
1 & \omega^{2} & \omega
\end{array}\right), \quad \omega \equiv e^{2 \pi i / 3}
$$

The above $M_{l}$ and $m_{D}$ imply that the symmetry basis $L_{i}$ also corresponds to the mass basis and only the righthanded fields need to be redefined. As a result, the neutrino mass matrix following from Eqs. (1) and (2), after the seesaw diagonalization [14], is already in the flavor basis and is given by

$$
\mathcal{M}_{\nu f}^{I}=m_{D} M_{R}^{-1} m_{D}^{T}=\frac{v^{2}}{M}\left(\begin{array}{ccc}
h_{1 D}^{2} & 0 & 0 \\
0 & 0 & h_{2 D} h_{3 D} \\
0 & h_{2 D} h_{3 D} & 0
\end{array}\right) .
$$

This has the same zero textures as obtained in [5] except that only two (instead of three) neutrinos are degenerate. As noted in [5], this texture by itself is not complete and one needs to modify it. For example, one can supersymmetrize the above scenario and use radiative corrections to split the degeneracy and obtain predictions for the mixing angles and masses as in [5].

Here we choose a different approach, introducing a triplet field $\Delta[15]$ transforming either as a $1^{\prime \prime}$ or as a $1^{\prime}$ under $A_{4}$, as in Table I. In the first case a small induced $\operatorname{VEV}\left\langle\Delta^{0}\right\rangle \equiv u$ for its neutral component leads to a type-II neutrino mass matrix contribution given as

$$
\mathcal{M}_{\nu}^{\mathrm{II}}=\left(\begin{array}{ccc}
0 & \lambda u & 0 \\
\lambda u & 0 & 0 \\
0 & 0 & \lambda^{\prime} u
\end{array}\right)
$$

where $\lambda, \lambda^{\prime}$ are two Yukawa couplings (another hybrid model based on $A_{4}$ and using both type-I and type-II contributions to neutrino masses has been considered in [16]). The total neutrino mass matrix is given by the sum of Eq. (4) and (5) and has the form

$$
\mathcal{M}_{\nu}=\left(\begin{array}{lll}
a & x & 0 \\
x & 0 & b \\
0 & b & y
\end{array}\right)
$$

where $a, b$ and $x, y$ refer to the type-I and type-II contributions, respectively. The above arguments provide a simple derivation of the two-zero texture classified as $B_{1}$ in Ref. [1]).

Alternatively, had the triplet been assigned to the $1^{\prime}$ representation of $A_{4}$ then we would have obtained

$$
\mathcal{M}_{\nu}=\left(\begin{array}{lll}
a & 0 & x \\
0 & y & b \\
x & b & 0
\end{array}\right)
$$

a texture classified as $B_{2}$ in [1]. One can modify the assignment of various $L_{i}$ fields among different singlet representations of $A_{4}$. This results either in one of the two above textures or in a texture that is not viable phenomenologically. Thus, the realization of the $A_{4}$ flavor symmetry proposed here leads to just two viable two-zero textures, which are quite predictive as we will show.

While the full $A_{4}$ symmetry is used in Eq. (1), the resulting two-zero textures follow essentially from a $Z_{3}$ subgroup of $A_{4}$ that remains unbroken by the vacuum structure in Eq. (2) [9]. This $Z_{3}$ is generated by $\left(1, z, z^{2}\right)$, $z^{3}=1$ with the leptons transforming as

$$
L_{i} \rightarrow Z_{i j}^{L} L_{j}, \quad\left(l_{R i}, \nu_{R i}\right) \rightarrow Z_{i j}^{R}\left(l_{R j}, \nu_{R j}\right),
$$

where $Z^{L}=\operatorname{diag}\left(1, \omega, \omega^{2}\right)$ and

$$
Z^{R}=\left(\begin{array}{lll}
0 & 0 & 1 \\
1 & 0 & 0 \\
0 & 1 & 0
\end{array}\right)
$$

Note that the fields which earlier transformed as triplets under $A_{4}$ are now put into a reducible representation of the $Z_{3}$ group. Let us now demand that $M_{l}, m_{D}$, and $M_{R}$ are invariant under the above defined $Z_{3}$. This implies

$$
Z^{L \dagger} M_{l} Z^{R}=M_{l} ; \quad Z^{L \dagger} m_{D} Z^{R}=m_{D} ; \quad Z_{R}^{T} M_{R} Z^{R}=M_{R} .
$$

It is straightforward to show that the above invariance implies that both $M_{l}$ and $m_{D}$ must have the form

$$
\left(\begin{array}{ccc}
X & X & X \\
A & \omega A & \omega^{2} A \\
B & \omega^{2} B & \omega B
\end{array}\right)
$$

The above form coincides with that obtained in Eq. (3) with proper identification of parameters. The right-handed neutrino mass matrix now has the following general form [17]

$$
\left(\begin{array}{lll}
M_{1} & M_{2} & M_{2} \\
M_{2} & M_{1} & M_{2} \\
M_{2} & M_{2} & M_{1}
\end{array}\right)
$$

In spite of this more complicated form, it is easy to see that the type-I contribution has exactly the same zero texture as in Eq. (4), which is therefore more general than its derivation through the seesaw mechanism used here. It simply follows from the $Z_{3}$ invariance of the effective neutrino mass matrix:

$$
Z^{L T} \mathcal{M}_{\nu} Z^{L}=\mathcal{M}_{\nu}
$$

irrespective of the underlying dynamics. For example, the same form would arise in a model without the right-handed neutrinos but containing a $Z_{3}$-singlet Higgs triplet with a nonzero VEV. As in the $A_{4}$ case, one can introduce a triplet $\Delta$ transforming as $\omega^{2}$ under $z$, and whose VEV will now break $Z_{3}$ to give the required two-zero texture as in Eq. (5).

We now turn to the phenomenological implications. The main feature of two-zero texture models, such as the ones 


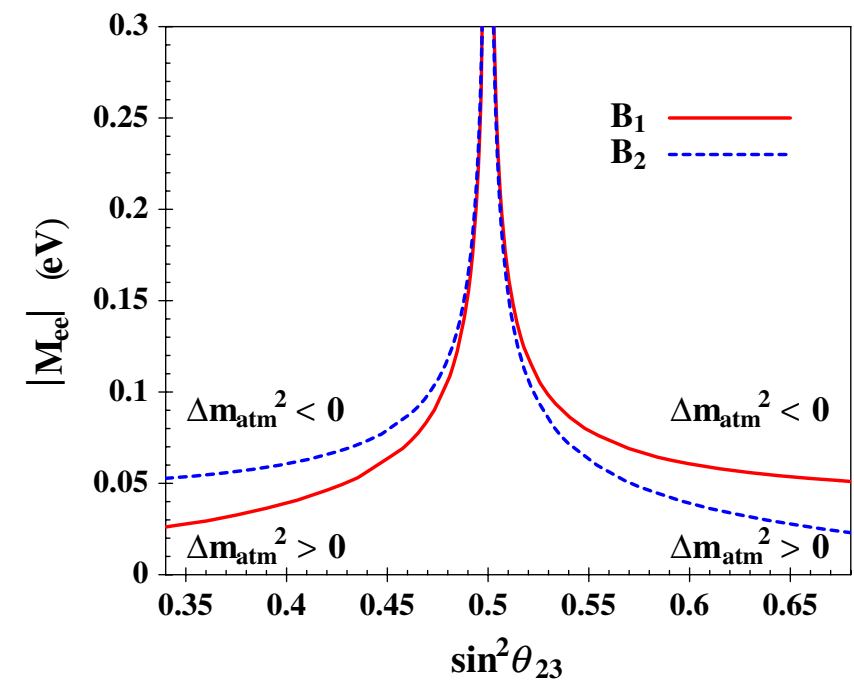

FIG. 1 (color online). Lower bound on neutrinoless double beta decay.

derived here, is their power in predicting the as yet undetermined neutrino parameters. Current neutrino oscillation experiments determine two mass splittings $\Delta m_{\mathrm{atm}}^{2}$ and $\Delta m_{\text {sol }}^{2}$ and the corresponding mixing angles $\theta_{12}$ and $\theta_{23}$, with some sensitivity on $\theta_{13}$, which is bounded [18]. The Dirac $C P$ phase will be probed in future oscillation experiments. Similarly, the absolute neutrino mass scale will be probed by future cosmological observations [19], tritium beta decays [20], and neutrinoless double beta decay experiments [21] with improved sensitivity. The latter will also shed light on the two Majorana $C P$ phases that are hard to test otherwise, as they do not affect lepton number conserving processes. The general $3 \times 3$ light neutrino mass matrix $\mathcal{M}_{\nu}$ in the flavor basis contains a priori nine independent real parameters, once the three unphysical phases associated with the charged lepton fields are removed. In contrast, in the proposed model all the above nine parameters are given in terms of only five unknowns. Hence the number of physical parameters characterizing the charged current weak interaction is reduced with respect to what is expected in the general case [15].

We now illustrate these predictions. We first consider the mass parameter characterizing neutrinoless double beta decay $\left|M_{e e}\right|$, which depends mainly on $\theta_{23}$, as illustrated in Fig. 1. A remarkable feature of our $A_{4}$ flavor symmetry model is that it implies the lower bound $\left|M_{e e}\right| \geqslant 0.03 \mathrm{eV}$, as seen in Fig. 1. This prediction correlates with the maximality of the atmospheric mixing angle and lies within the range of planned experiments. The bound hardly depends on other parameters. For example, in contrast to Ref. [10], it shows no strong dependence with the value of the relevant Majorana phase. This follows from the more stringent lower bound on the lightest neutrino mass obtained in the present model. We note that $\left|M_{e e}\right|$ has, however, some dependence on the value of $\Delta m_{\text {atm }}^{2}$ and the bound corresponds to $\Delta m_{\mathrm{atm}}^{2}=2 \times 10^{-3} \mathrm{eV}^{2}$.

We now turn to the predictions for $C P$ violation and the parameter $\delta$.

As seen in Fig. 2, for both the $B_{1}$ (left panel) and $B_{2}$ cases (right panel), our model predicts the near maximality of the $C P$ violation in neutrino oscillations. The predicted $C P$ violating parameter $\delta$ depends mainly on $\theta_{13}$, which is currently bounded only by oscillation data [18].

The rephasing invariant magnitude $|J|$ of $C P$ violation in neutrino oscillations is defined as

$$
J=\operatorname{Im}\left[K_{11} K_{22} K_{12}^{*} K_{21}^{*}\right]=s_{12} s_{23} s_{13} c_{12} c_{23} c_{13}^{2} \sin \delta,
$$

where $K_{i j}$ are the elements of the leptonic mixing matrix. As seen in Fig. 3, which holds for both $B 1$ and $B 2$ models, one finds that $|J|$ is directly correlated with the value of $\sin ^{2} \theta_{13}$, to be probed in the next generation of high sensitivity neutrino oscillation experiments such as Double

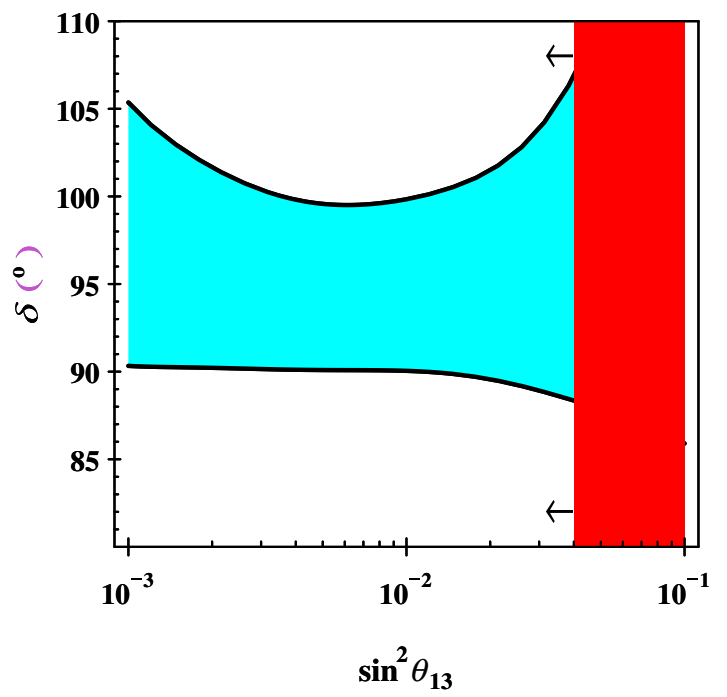

FIG. 2 (color online). Near-maximal $C P$ violation in neutrino oscillations. 


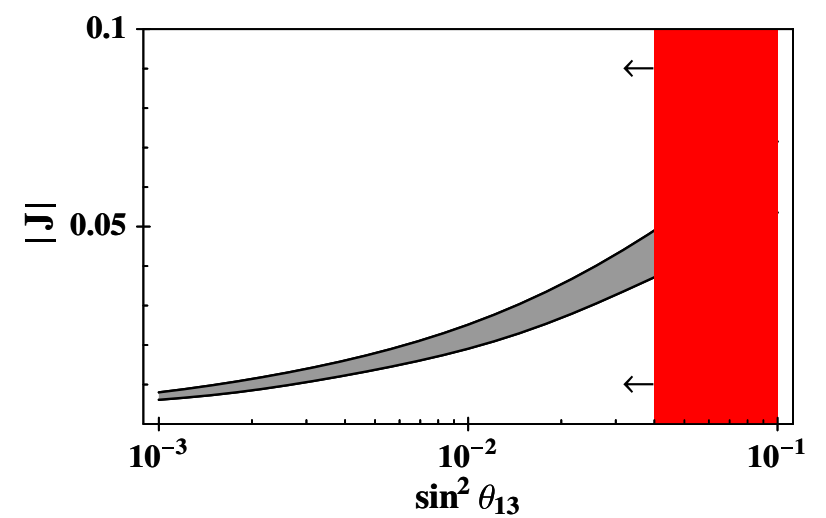

FIG. 3 (color online). $C P$ violation in neutrino oscillations versus $\sin ^{2} \theta_{13}$.

Chooz. The width of the band reflects the current uncertainties in the neutrino oscillation parameters [18].

The above predictions based on the tree level texture of $\mathcal{M}_{\nu}$ are expected to change very little as a result of radiative corrections. The $Z_{3}$ symmetry giving tree level texture in Eq. (4) protects it to all orders. Since $Z_{3}$ gets broken by the triplet VEV, finite radiative corrections involving triplet Higgs fields modify tree level zeros. However, these are suppressed at least by the square of the Yukawa couplings times the loop factor and hence are small.

In summary, here we have proposed an $A_{4}$ flavor symmetry for leptons that leads to a near-maximal $C P$ phase $\delta$ and correlates the invariant measure of $C P$ violation in neutrino oscillations with the magnitude of $\sin ^{2} \theta_{13}$ to be probed in future neutrino oscillation experiments. Moreover, it implies a lower bound $\left|M_{e e}\right| \gtrsim 0.03 \mathrm{eV}$ for the mass parameter characterizing neutrinoless double beta decay, also accessible to planned experiments. All these features already emerge from an effective $Z_{3}$ invariance of the larger $A_{4}$ symmetry. However, the structure of $M_{R}$ is different in the $A_{4}$ model and the effective $Z_{3}$ model. Hence, for example, some phenomenological details related to leptogenesis could be different. These issues will be taken up elsewhere.

This work was supported by MEC Grants No. FPA200501269, No. SAB2005-160 (A. J.), and No. FPA200525348-E, by Generalitat Valenciana ACOMP06/154, by European Commission Contracts No. MRTN-CT-2004503369 and No. ILIAS/N6 RII3-CT-2004-506222. *mahirsch@ific.uv.es

†anjan.@prl.res.in

"satoru@ific.uv.es

§valle@ific.uv.es

[1] P. H. Frampton, S.L. Glashow, and D. Marfatia, Phys. Lett. B 536, 79 (2002).

[2] S. Dev, S. Kumar, S. Verma, and S. Gupta, Phys. Rev. D 76, 013002 (2007); Z.z. Xing, Phys. Lett. B 539, 85 (2002); 530, 159 (2002); A. Kageyama, S. Kaneko, N. Shimoyama, and M. Tanimoto, Phys. Lett. B 538, 96 (2002).

[3] W. Grimus, A. S. Joshipura, L. Lavoura, and M. Tanimoto, Eur. Phys. J. C 36, 227 (2004).

[4] E. Ma and G. Rajasekaran, Phys. Rev. D 64, 113012 (2001).

[5] K. S. Babu, E. Ma, and J. W. F. Valle, Phys. Lett. B 552, 207 (2003).

[6] P. F. Harrison, D. H. Perkins, and W. G. Scott, Phys. Lett. B 530, 167 (2002).

[7] S.F. King and M. Malinsky, Phys. Lett. B 645, 351 (2007).

[8] E. Ma, Phys. Rev. D 73, 057304 (2006).

[9] X.-G. He, Y.-Y. Keum, and R. R. Volkas, J. High Energy Phys. 04 (2006) 039.

[10] M. Hirsch, A. Villanova del Moral, J. W. F. Valle, and E. Ma, Phys. Rev. D 72, 091301 (2005).

[11] G. Altarelli and F. Feruglio, Nucl. Phys. B741, 215 (2006).

[12] E. Ma, Phys. Rev. D 70, 031901 (2004); Mod. Phys. Lett. A17, 627 (2002).

[13] E. Ma, Mod. Phys. Lett. A21, 2931 (2006).

[14] For a recent seesaw review see J. W. F. Valle, J. Phys. Conf. Ser. 53, 473 (2006), based on lectures at the Corfu Summer Institute on Elementary Particle Physics 2005.

[15] J. Schechter and J. W.F. Valle, Phys. Rev. D 22, 2227 (1980); 25, 774 (1982).

[16] S.-L. Chen, M. Frigerio, and E. Ma, Nucl. Phys. B724, 423 (2005).

[17] This more general form would arise in a model containing an $S U(2)_{L}$ singlet but $A_{4}$ triplet Higgs field $\eta$ with a $Z_{3}$-preserving $\mathrm{VEV}$.

[18] For a review see M. Maltoni, T. Schwetz, M. A. Tortola, and J. W. F. Valle, New J. Phys. 6, 122 (2004); The last appendix in arXiv:hep-ph/0405172 (v6) includes the latest neutrino oscillation data of September 2007, as well as references to previous analyses.

[19] J. Lesgourgues and S. Pastor, Phys. Rep. 429, 307 (2006).

[20] G. Drexlin (KATRIN Collaboration), Nucl. Phys. B, Proc. Suppl. 145, 263 (2005).

[21] See invited talks at Neutrino 2006, M. Hirsch, arXiv:hepph/0609146; S. R. Elliott, arXiv:nucl-ex/0609024. 